\title{
Content Aware Retargeting of Stego Images
}

\author{
K. Thilagam \\ Ph. D. Research Scholar, \\ Karpagam University, \\ Coimbatore, \\ India
}

\author{
S. Karthikeyan, \\ Ph. D. Assistant Professor, \\ Department of IT, \\ College of Applied Sciences, \\ Sohar, Sultanate of Oman
}

\begin{abstract}
Images play a vital role in steganography, as cover media to hide secret messages. Retargeting becomes mandatory while transmitting image in a heterogeneous network because sender and recipient may not have display devices of same resolution. Resizing images by scaling or cropping also lead to loss of visually important features. In steganography, resizing, compressing, and changing the color or format of stego images would result in loss of hidden information. In this paper we attempt to retarget stego images in a content preserving manner using a modified seam carving technique called PSC. Content in this context refers to the visually salient features in the cover image as well as the secret message hidden within it. The cover image is first subjected to PSC to extract as many seams leaving the segments containing ROIs intact. Based on the size of secret message segment(s) containing the ROIs are selected to encode the secret message and is transmitted to the receiver. The seams are also encoded separately in the reverse order of their extraction, so that on the receiver end the receiver would download all the image segments containing the ROIs and as many seams as required to constitute an image that fits into the target device. The retargeted image contains the secret message intact which could be decoded by the receiver.
\end{abstract}

\section{General Terms}

Image Processing, Information security

Keywords

PSC-Piecewise Seam Carving, Image Resizing, Retargeting, Steganography.

\section{INTRODUCTION}

Steganography [8] is an art / science of hiding information within a cover media (carrier), so that the innocent viewers are unaware of a secret message hidden within it. The cover media shall be a text file, sound clip, image file etc., among which images are the widely used. The image file, in which we hide the Secret_msg, is called the Carrier_img. The Secret_msg may optionally be encrypted using the Crypt_key. The resultant file is the Stego_img.

Stego_img=Carrier_img + Secret_msg+(optionally)Crypt_key.

Let $F_{e}$ be the steganographic function that encrypts and embeds the Secret_msg within the Carrier_img. The Stego_img is then sent to the recipient, who can extract the Secret_msg\# and the Carrier_img\# from it using the inverse steganographic function $\mathrm{F}_{\mathrm{e}}^{-1}$. Also if the message is encrypted, decryption is done using the inverse of Crypt_key called the Decrypt key. Fig.1 represents the Process of Steganography.

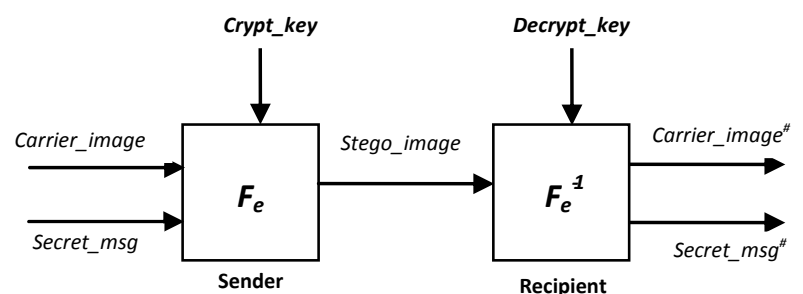

Fig 1: Process of Steganography.

In steganography the sender and receiver may not use hardware of same architecture. The stego_img containing the secret_msg, is transmitted to the recipient and is displayed in a different display device. Diversity of display devices available in the market provide a choice for high quality display of multimedia contents, but pose the problem of integrity. Varying resolution of display devices requires image retargeting, altering the size of the image to fit into the target device. Resizing stego images, using lossy compression on them, changing the color or format of the images all lead to loss of information hidden within it. Therefore such techniques are used to defeat steganography. We propose a method for retargeting cover images leaving the visually salient content and the hidden message intact.

\section{BACKGROUND}

It is desirable to retain the visually salient contents (ROIs) of the image intact while retargeting the images. The state-of-art methods like scaling, cropping and warping are content unaware but widely used because of their simplicity, when the visual impact of the image is not much important. Seam carving [1] which has recently gained popularity is a good choice for content aware image retargeting.

\subsection{Overview of Seam Carving}

Seam carving [1] is a content aware image resizing algorithm proposed by Ariel Samier and Shai Avidhan. For image size reduction this algorithm iteratively removes optimal, monotonic, 8-connected path of low energy pixels that contribute less to the visual impact of the image, thereby preserving the visually salient contents. This path of pixels is termed as seam. Similarly for image magnification optimal seams are computed and duplicated. Seams are carved/inserted in both directions, or in one direction (horizontally / vertically) so as to change the aspect ratio of the image. This algorithm contributes to the generation of multisize images, image size reduction / magnification so as to fit into any target display device and also for removal of an object from the image.

It always removes or inserts low energy pixels until the desired image size is achieved, and hence the ROI's with 
relatively low energy cannot sustain from being carved out or distorted. Denser regions of interest (ROI) in the image and sometimes the orientation of the image make seams unavoidably bypass the important regions thereby distorting it. It cannot preserve the geometric shapes. It also has a performance lacuna, due to extensive computation for pixelby-pixel manipulation to recalculate the energy map after every seam is removed / inserted. Several optimizations were done to improve Seam Carving. Parallel algorithms, graph cuts, are used to improve their computational efficiency, Forward energy as an alternative to Backward energy, Image distance measures, Dominant color descriptors and Fuzzy based techniques are used to improve their visual effects. Seam carving, in combination with other traditional methods were tried to improve its efficiency and to minimize its negative impacts.

\subsection{Problem in retargeting stego images}

The stego image also contains the secret information hidden within it apart from the visual contents. However the pixel removal/insertion technique of Seam carving would result in loss (removal) of pixels coded with secret message or would jumble the message by inserting pixels in between pixels coded with secret message and make it difficult to interpret. This would spoil the main aim of steganography. Moreover resizing stego images, using lossy compression on them, changing the color or format of the images all lead to loss of information hidden within it. Therefore such techniques are used to defeat steganography. So no attempts were made to resize stego images. In our research we attempt to resize the stego images with an objective to preserve both the visual saliency of the carrier image and also the secret information it carries.

Our contributions are stated below

- Apply piecewise approach of seam carving to resize the image to be transmitted so as to reduce the bandwidth requirement.

- Enables the recipient to download image based on his device requirement (Retargeted image)

- Preserve the secret message hidden within the image, received correctly on the recipient side and also to leave the visually salient content intact while retargeting.

The methods applied in steganography and retargeting are explained in section 3. In Section 4 we discuss upon the results produced and conclude in the section 5 .

\section{PROPOSED METHOD}

The carrier image is resized using either SC or PSC. This result in two components: i) resized image contains the ROIs intact, and ii) a set of seams. The resized image is used as a cover image for hiding secret information in steganography. Any suitable steganographic method shall be adopted to hide the secret message. Optionally the secret message shall be encrypted before being hidden, using a crptying key, to enhance security of the message. This stego image is then encoded for transmission from the sender side. The seams extracted are stored in a seam vector in the order of their extraction along with its position and color information and are encoded after the stego image, using the codec proposed by [2] in the reverse order of their extraction. The receiver would decode the entire stego image losslessly and apply inverse steganographic function to extract the hidden information without any loss. If the message is encrypted, then it is decrypted to reveal the exact message. The seams that are received after the core segments shall be decoded as per the requirement of the target device. Fig.2 shows the diagrammatic representation of the proposed system.

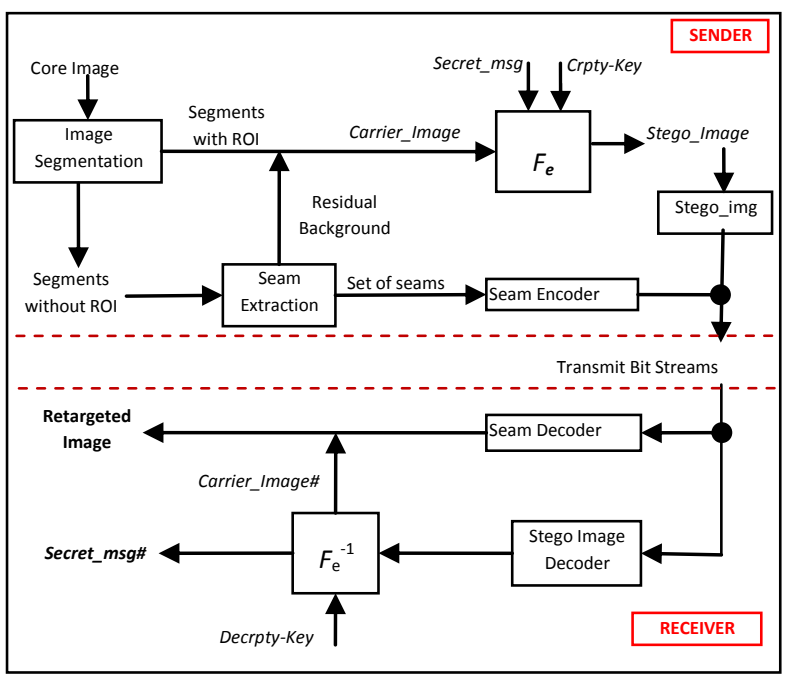

Fig 2: Block Diagram of the Proposed Method

\subsection{Piecewise/Proportional seam carving:}

$\mathrm{PSC}[3]$ is a interactive content aware image resizing technique which segments the image based on the extent and orientation of the ROIs and apply seam carving to each segment in varied proportion, so as to preserve the ROIs even those with relatively low energy. This piecewise approach of seam carving help either to disallow or carve only less no of seams in some segments (containing the ROIs) where as other segments shall be carved extensively. This technique is optimized by a semi automated interface that use a significance map[4], to automatically identify the visually prominent regions of an image, in addition with user interaction to select the low energy ROIs. Significance map is computed as a product of the gradient magnitude[1] and saliency measure[5]. The boundaries of the ROIs are used as the segment limits and segmentation is just a virtual partitioning to limit the seams bypassing the ROIs. We apply the same computation techniques in [1] but the energy function is modified to consider the energy inserted[6,7] into the image while removing the low energy seams. Apart from seams being monotonic and 8-connected, an additional constraint is imposed on seam that it must lay within the same segment limits.

PSC has several advantages over the parent method which are stated below. It can strictly restrict seams bypassing ROIs, which is not possible in the parent method. It can even preserve the ROIs that have relatively low energy. The computation complexity of PSC is far less than that of SC, because the energy map recalculation after removal of every seam, involves only the pixels of a particular segment within which the seam lies, instead of the entire image[1]. And shifting the pixels to the right of the seam to replace the removed seam is also performed only within the segment in which the seam is removed. Fig. 3 shows a comparison of the resizing results obtained by various methods and Fig.4 compares the time taken to resize images using PSC with SC and the Fast Multioperator technique. 


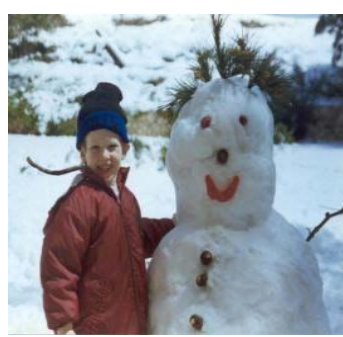

(a)

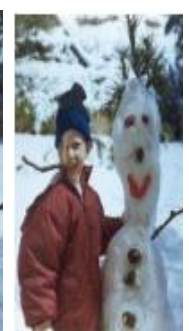

(b)

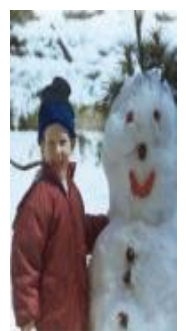

(c)

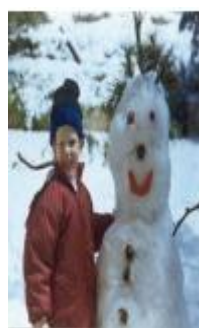

(d)

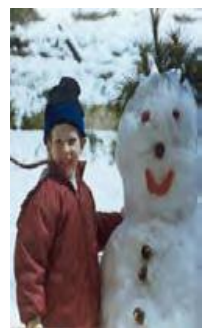

(e)

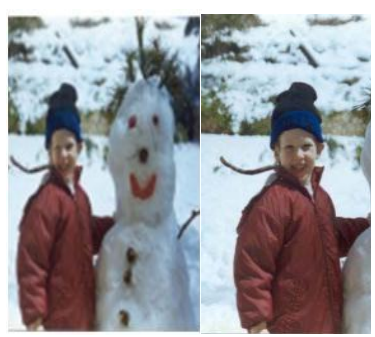

(g)

Fig.3 (a) Original Image, (b) Seam Carving, (c) Multioperator, (d) Seam Carving and Scaling, (e) Fast Multioperator, (f) Piecewise Seam Carving, (g)Cropping

Moreover the independent application of SC to each segment and availability of modern hardware with multicore processors in the market may profit from parallel algorithms. Using linear algorithms the time taken to resize the image by PSC is the sum of time taken to resize each segment. When parallel programming is used and if there are as many processors as the number of segments, then the time of resizing is the maximum among the time taken to resize individual segments, which obviously will be less than that of SC. When sufficient seams are removed the sub matrices constituting the segments are merged into the final matrix that forms the resized image. Several optimizations were made to improve the performance efficiency of SC, which could also be generalized to PSC.
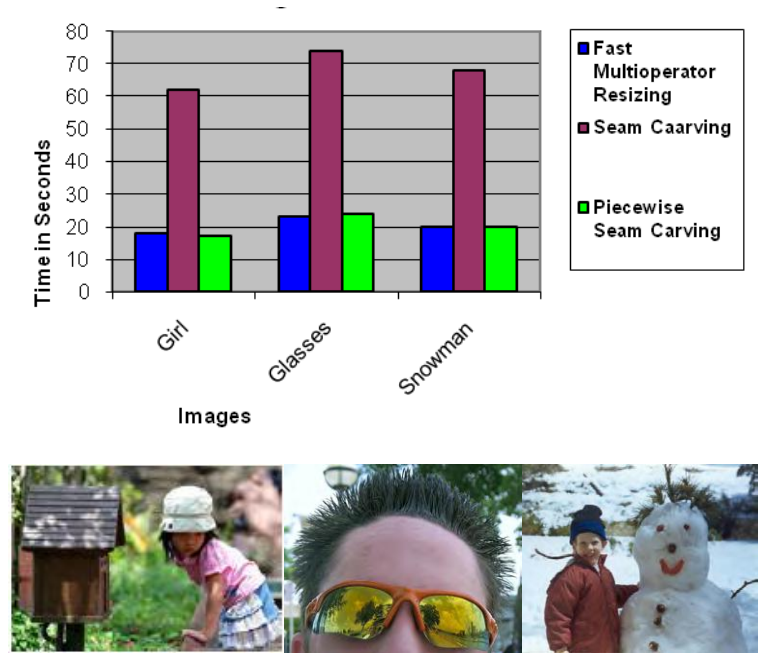

Fig.4 Comparison of time taken to resize Images

\subsection{SLSB Steganography}

The resized image is used as cover media to hide secret message. Several steganographic algorithms that work either in transform domain or spatial domain shall be used for hiding. Algorithms in spatial domain are simpler and fast compared to algorithms in transform domain which are more complex and robust. We apply the simplest and best known form of steganography in spatial domain called the Least Significant Bit (LSB) steganography in which one or lower order bit planes are replaced with the secret code bits. As we use color image as the cover media, LSB of any one dominant color component (Red Green Blue) shall be used to hide the secret message $[9,10]$. Selected Least Significant Bit(SLSB)[9] Steganography is a variant of LSB that selects the dominant color using the Sample Pairs Analysis and applies filtering techniques for selecting pixels for encoding the secret message within the LSBs of the selected color. As stated by the probability theory of random experiment, atmost $50 \%$ of pixels used for message hiding shall switch its original value from 0 to 1 or 1 to 0 . An LSB matching is used to minimize variation between the original and steganographic pixel. The SNR (signal-to-noise ratio) values are high and the Mean Square Error (MSE) values are low for SLSB steganography. The higher the SNR value, reduced is the rate of detection of hidden message. Table.1 shows the results obtained[9] by familiar steganographic methods for hiding 31 bytes of text information within an image of size 7.86 kilobytes.

Table.1. Comparision of MSE,SNR and PSNR values

\begin{tabular}{|l|r|r|r|}
\hline \multicolumn{1}{|c|}{ Tool } & \multicolumn{1}{c|}{ MSE } & \multicolumn{1}{c|}{ SNR } & \multicolumn{1}{c|}{ PSNR } \\
\hline Original image & $\mathbf{0 , 0 0 0}$ & $\mathbf{0 , 0}$ & $\mathbf{0 , 0}$ \\
\hline SLSB (1 bit/pixel) & 0,020 & 6,7 & 2,0 \\
\hline SLSB (2 bits/pixel) & 0,043 & 3,2 & 9,9 \\
\hline SLSB (3 bits/pixel) & 0,137 & 1,0 & 3,1 \\
\hline Contraband & 0,872 & 136124,5 & 415146,6 \\
\hline Data Privacy Tools & 13,760 & 7530,1 & 22965,0 \\
\hline Digital Invisible Ink & 0,947 & 135973,3 & 414685,4 \\
Toolkit & 90,996 & 1713,9 & 5227,1 \\
\hline EikonaMark & 4,500 & 33278,5 & 101491,3 \\
\hline Hermetic Stego & 0,477 & 134339,1 & 409701,5 \\
\hline Hide4PGP & 0,665 & 134508,4 & 410218,1 \\
\hline Hide In Picture & 3,007 & 42830,9 & 130623,8 \\
\hline Invisible Secrets & 9,141 & 11427,0 & 34849,6 \\
\hline JPHS & 0,320 & 348621,5 & 1063210,6 \\
\hline Steghide & 0,025 & 2559393,7 & 7805527,2 \\
\hline S-tools & 0,947 & 135563,4 & 413435,6 \\
\hline wbStego & 1,101 & 102060,9 & 311260,8 \\
\hline White Noise Storm & & \\
\hline
\end{tabular}

In the transform domain, a maximum SNR value is achieved for the color component that contributes most energy in the wavelet domain [10]. A wavelet transform shall be applied to each of the color component independently to select a color that contributes much energy (or high SNR value). Moreover, regardless of the color component selected, the second correlation coefficient between the wavelet coefficients of the 
original image and the stego image is almost 1.0. This means that the message embedded in the LSB planes of any color do not affect the wavelet coefficients. This proves the applicability of wavelet based encoding techniques for image transmission without damaging the hidden message.

The stego image that is encoded losslessly is transmitted to the receiver and is decoded by the receiver. The end of file marker is used as a trigger to invoke the inverse steganographic function. Prior to it the LSBs of the selected color in the stego image are extracted and stored in a separate matrix. Subsequently the seams are downloaded to retarget the image of required size, so that the innocent viewer receiving the image will not suspect the presence of secret message hidden within it. Codec used to encode and decode the seam is explained in the next subsection. Later the LSBs are decoded to reveal the secret message. When the secret message is encrypted, it shall be decrypted by applying the inverse crypting function.

\subsection{Seam Codec}

Seams are extracted in both directions subsequently and are stored in two seam vectors SVR and SVC, SVR contains the row seams and SVC contains the column seams. The color and position information of each pixel constituting the seam are stores in the seam vector. Let us assume that the horizontal and vertical resolutions of the image are $2^{\mathrm{N}}$. As the seams are monotonic (one in each row), to represent the pixel positions of a vertical seam, it is sufficient to code only the $\mathrm{x}$ coordinate value (y-coordinate value continuously increases from 1 to $2^{\mathrm{N}}$ ). Similarly for a horizontal seam only the $y$ coordinate value shall be represented. The absolute position of the first pixel of each seam has to be encoded. As seams are 8connected the position of rest of the pixels in the seam is coded relative to the previous pixel, as a difference in its $\mathrm{x} / \mathrm{y}$ coordinate value which can take one of the three possibilities $(-1,0,1)$. An adaptive arithmetic coding algorithm with $2^{\mathrm{N}+1}$ symbols and 3 symbols are used respectively to encode the first seam pixel and rest of the pixels, for transmission. At the beginning of each seam one bit is used to indicate if it is a vertical or horizontal seam. The color of the seam pixels can be coded similar to position information. For a 24 bit RGB color image, the absolute color of the first pixel is coded, and the difference in color is coded for the rest of the pixels which is in the range (-255 to 255$)$. So we use an adaptive arithmetic coding algorithm with $2^{9}-1$ symbols. But most of the color differences have small value, and no secret information coded within the seam pixels, so a lossy quantization shall be applied on seam pixels.

As many number of seams as required can be computed but the first seam is the most optimal or least significant one than that of the remaining. Each new seam has higher significance than the ones extracted previously. A seam ordering has to be maintained so that while encoding in the codec for transmission the order shall be reversed. The last seam computed is the first one to be received / decoded in the receiver side. Vertical and horizontal seams are alternatively encoded and transmitted. The receiver can download as many seams based on the resolution of his display device and add it up to the decoded core image. Number of vertical and horizontal seams required may not be same. If seam requirement is satisfied along one direction, then further seam in that direction shall be discarded only adding seams in the other direction. When the target resolution is greater than that of the original image then seams has to be duplicated to enlarge the image.
An alternative method shall be applied if the receiver hardware is capable of running SC algorithm locally; only the core image containing the hidden message shall be transmitted to the receiver and decoded to extract the secret message. The seams extracted at the sender side are of not much importance and shall be discarded. At the receiver end a set of seams shall be computed on the spot and added to the image to further enlarge the image to fit target device resolution. This has the advantage of minimizing transmission requirements, but the image quality (originality of image) degrades with the increase in target resolutions when number of seams added is more.

\section{RESULTS AND DISCUSSION}

In the example below Fig 5.a is the original cover image used to hide the text file (Abstract.txt) that contains the abstract of this paper. Fig 5.b shows 25 vertical seams that are extracted and stored in the seam vector. Fig 5.c is the resized image after 25 seams extracted from it. Fig 5.d is the stego image which contains the message hidden within it. Fig 5.e shows the simulation of hidden pixels. Fig 5.f shows the seams being inserted subsequently after the stego image is received in the receiver side.

In [11] attempts were made to resize the secret image (secret message) using seam carving, before it is hidden within the carrier image. No attempts were made to resize the cover media to fit the target device. The main advantage of our method is the difficulty to detect the hidden message by recipients other than the intended recipient. Only the intended recipient would trigger the inverse steganographic functions to extract the encoded LSBs, immediately after the resized stego image is received. For other recipient the seams are downloaded subsequently and added to the cover image thereby jumbling the message. Intruders trying to extract the message after the entire retargeted image is obtained would find it difficult to interpret. Using LSB steganography has the advantage of less transmission overheads as only the cover image and as many seams as required shall be transmitted and decoded in the receiver side, and the original image is not needed to reconstruct the secret message. If the receiver is capable of running seam carving algorithm locally a set of seams can be generated on the spot and added to this image (seam insertion) to enlarge the image further to fit the target device but this would degrade the image quality.

The choice of color component is the next issue for optimal message hiding. The color component that contributes much to the image energy is chosen, because it is likely to form the ROIs expected to be retained while retargeting. Also note in the simulation map in Fig.5.e the pixels used for hiding the message forms the ROIs of an image. This insists the idea of coding the secret message within the core segments containing the ROIs. Results show that the color which contributes much to the image energy has a high SNR value, i.e., reduce the chance of detection of hidden message. The larger color component is chosen so as to increase the payload of steganography. SLSB is immune to visual attacks, histogram comparison and statistical attacks, so the message hidden within it is not easily detectable.

Larger the size/number of ROIs in the image less is the number of seams that can be computed without distorting them. Therefore the retarget ratio is low. The stego_img shall even be compressed losslessly, but the problem is the choice of compression technique which would not destroy the hidden message. 


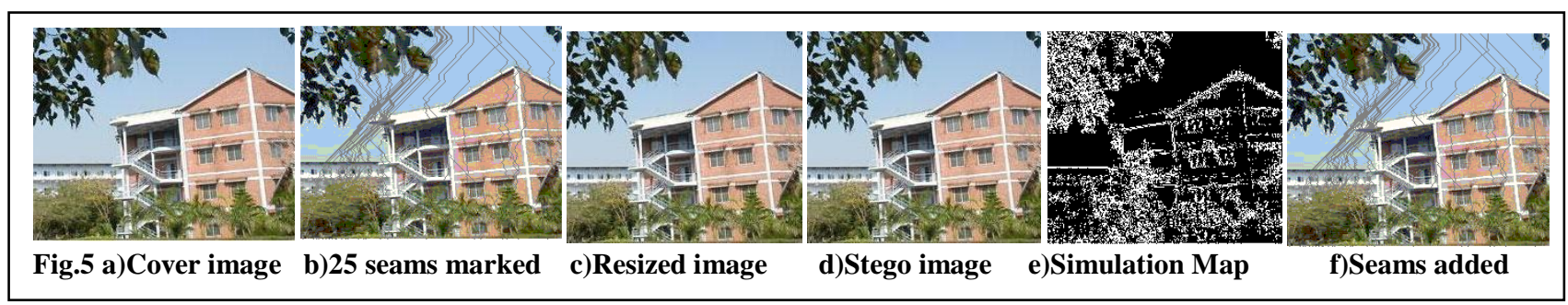

\section{CONCLUSION AND FUTURE SCOPE}

Heterogeneity of display devices used in communication makes image retargeting mandatory. Steganography proves an efficient method for conveying secret information and sometimes the hidden message may have connection to the visual contents stressing the need for content aware retargeting. This method appears to be very good in retargeting images, preserving the ROIs of user choice, and also the hidden message.

Applying lossy compressions on stego images would destroy the hidden message but a loss less compression shall be tried to further reduce transmission requirements. At the sender side seam extraction from each segment shall be parallelized as segments are processed independent of the other. Similarly at the receiver end, the LSBs are extracted from the cover media, immediately followed by seam extraction, which can be parallelized with secret message decoding process at the background, to reduce the downloading time. Several steganographic methods available shall be used to compare and evaluate the efficiency of this method. Alternate steganographic approaches capable of taking advantage of segmented approach of PSC, shall be tried so that difficulty in predicting the segment limits would reduce the chance of detecting information by steg analysis.

\section{REFERENCES}

[1] Avidan. S and Shamir A., "Seam carving for contentaware image resizing”. ACM SIGGRAPH, 26(3), (2007).

[2] Nguyen Thi Nhat Anh, Wenxian Yang. and Jianfei Cai, "Seam Carving Extension: a Compression Perspective", ACM Multimedia, 825-828, (2009).

[3] K Thilagam and S Karthikeyan, "Optimized Image Resizing using Piecewise Seam Carving”, International Journal of Computer Applications 42(14):24-30, (2012).

[4] Wang Y.-S, Tai C.-L, Sorkine O. and Lee T.-Y, "Optimized scale-and-stretch for image resizing". ACM Transactions on Graphics, 27(5), Article: 118, (2008).

[5] Itti,L., Koch,C. and Niebur, E, “ A Model of saliency based visual attention for rapid scene analysis", in IEEE Transactions on Pattern Analysis and Machine Intelligence, 20,1254-1259, (1998).

[6] Rubinstein M., Shamir A. and Avidan S, "Improved seam carving for video etargeting", ACM Transactions on Graphics, 27(3), Article16, (2008).
[7] Wei-Ming Dong, Guan-Bo Bao, Xiao-Peng Zhang and Jean-Claude Paul, "Fast Multi-Operator Image Resizing and Evaluation", Journal of Computer Science and Technology, 27(1), 121-13, (2012).

[8] Arvind Kumar and Km. Pooja, "Steganography- A Data Hiding Technique", International Journal of Computer Applications, 9(7), 19-23, (2010).

[9] Juan José Roque and Jesús María Minguet, "SLSB: Improving the Steganographic Algorithm LSB", 7th International Workshop on Security in Information Systems, 57-66, (2009).

[10] Abdullah and A. Basuhail, "Color Component Selection for Best Construction of Stego Images Using Wavelets", the Fifth Saudi Technical Conference and Exhibition, (2009).

[11] Toony, Z. and Jamzad, M. "A Novel Image Hiding Scheme Using Content Aware Seam Carving Method", in International Conference on Availability, Reliability, and Security, (2010).

\section{AUTHORS PROFILE}

Thilagam.K. has completed MCA., M.Phil., and currently pursing Ph.D., in Computer Science at Karpagam University, Coimbatore. She has been working as lecturer in various colleges and at present she is with Karpagam University as an Assistant Professor. She has presented more than 12 papers in national/International conferences and journals. Her research interests include digital image processing, data compression and steganography.

Karthikeyan S. received the Ph.D Degree in Computer Science and Engineering from Alagappa University, Karaikudi in 2008. He is working as a Professor and Director in School of Computer Science and Applications, Karpagam Univeristy, Coimbatore. At present he is in deputation and working as Assistant Professor in Information Technology, College of Applied Sciences, Sohar, Sultanate of Oman. He has published more than 17 papers in National/International Journals. His research interests include Cryptography and Network Security. 